\title{
VIBRATION AND NOISE IDENTIFICATION OF A COMMERCIAL REFRIGERATOR
}

\author{
Wolfgang Alois Hörtnagel ${ }^{1}$, Stefan Plagg ${ }^{2}$ and Fadi Dohnal ${ }^{1}$ \\ ${ }^{1}$ UMIT - Private University for Health Sciences, Medical Informatics and Technology \\ Linker Iselweg 21, 9900 Lienz, Austria \\ wolfgang.hoertnagel@umit.at,www.umit.at $/ \mathrm{melz}$ \\ ${ }^{2}$ Liebherr-Hausgeräte Lienz GmbH \\ Dr.-Hans-Liebherr-Straße 1, 9900 Lienz, Austria \\ www.liebherr.at
}

Key words: Applications, Computing Methods, System Identification, OTPA

\begin{abstract}
The vibration transmission to the cooled goods within a refrigerator is a usually neglected field of interest. Previous studies however show that biochemical properties can be affected by continuous exposure to vibration. Optimal storage conditions necessitate protection against UV light and vibration to prevent the cooled good's quality being degraded by outside factors. Using operational transfer path analysis, we investigate different excitation sources that affect a commercial refrigerator and quantify the spectral input-distributions for all potential sources. Environmental influences, operational conditions and human interactions with both the refrigerator directly as well as indirectly via the environment were considered. Based on previous works, we developed a robust algorithm for the estimation of the varying transmission paths within the refrigerator and the close environment. Utilizing the measured and calculated data we investigate optimizations in design. We identified and charactarized the impact of different vibration-sources and used deflection shapes of the system and the results from the TPA to identify areas of interest. Finally, we investigate measures to shift resonance frequencies into spectral ranges with less excitation and to introduce additional damping.
\end{abstract}

\section{Introduction}

Cooling devices have been the subject of numerous studies. The vibroacoustic analysis usually $[1,2,3,4]$ focusses on the psychoacoustics and the impact of engineering on the emission of vibrations in the audible frequency ranges. While the propagation of vibrations is also considered [5,6], these studies tend to narrowly focus on the surrounding of one active component, e.g., a compressor or a fan.

Continuous exposure to vibration was shown [7] to affect the biochemical properties of stored wine and the changes became more significant at higher vibration levels. Optimal storage conditions necessitate [8] protection against UV light and vibration to prevent the reduction of aromatic components and the formation of undesirable flavor and taste. While some goods [9] tend to be robust against these outside factors others [10] are particularly susceptible to these effects. Human cells are vulnerable to cold environments and the coupling of cold storage and vibration leads to membrane rupture and cell death.

In this paper, we will investigate different sources for the vibrations inside the commercial refrigerator 
shown in figure 1 and propose improvements to reduce the exposure of the cooled goods within.
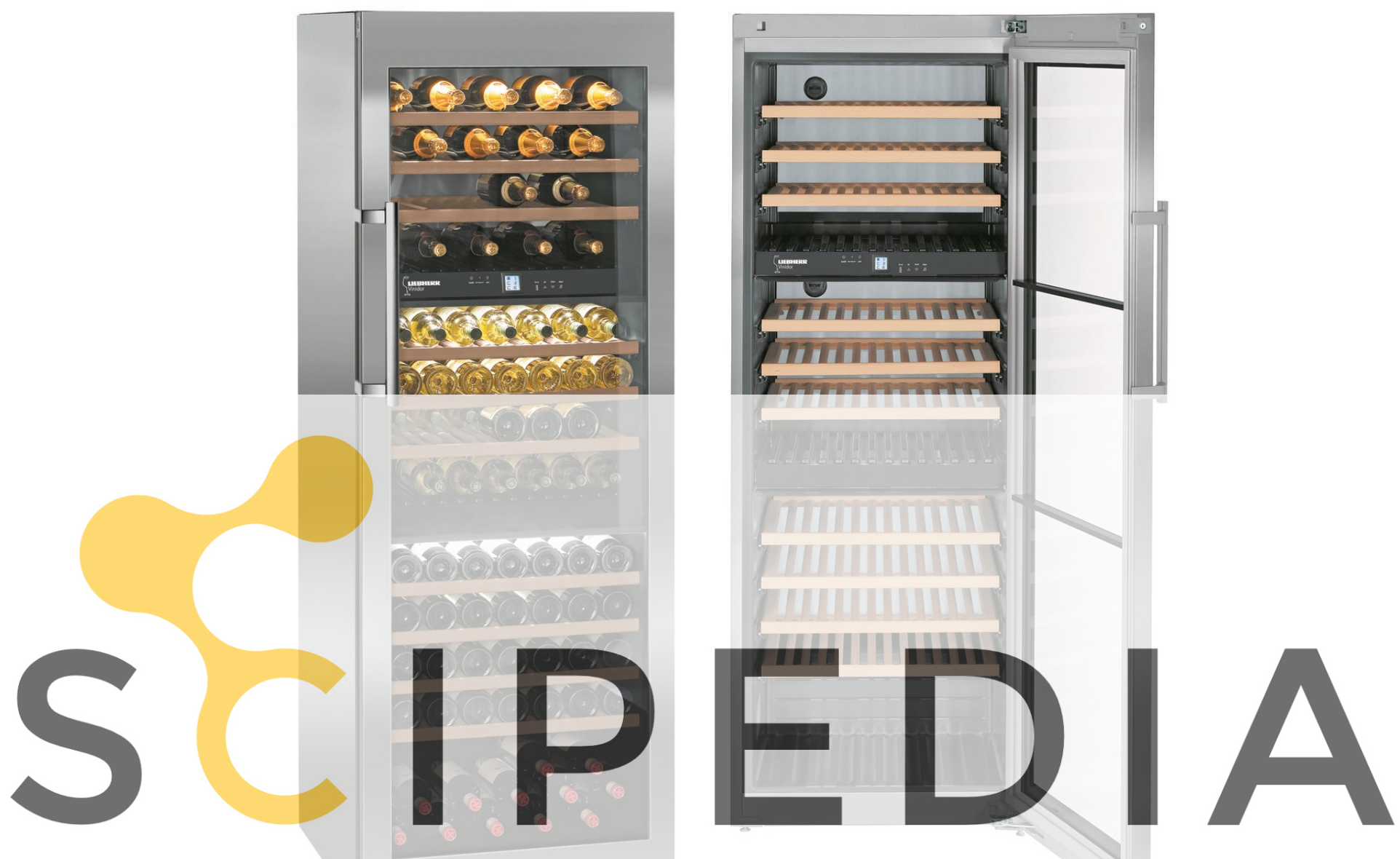

Register for free at https//www.scipedia.com to download the version without the watermark

Figure 1: The investigated device is a Liebherr WTes 5872 Vinidor. This multi-temperature wine cabinet has a frame made of stainless steel, a glaced door and wooden grid shelves. Up to 178 bottles can be stored in 13 storage shelves.

Table 1: Technical data for the WTes 5872 Vinidor

\begin{tabular}{|l|c|}
\hline Product dimensions (H/W/D) & $192.0 / 70.0 / 74.2 \mathrm{~cm}$ \\
\hline Weight (without packaging) & $134.00 \mathrm{~kg}$ \\
\hline Net capacity, total & 4961 \\
\hline Maximum number of 0.75 1 Bordeaux bottles & 178 \\
\hline Noise level & $38 \mathrm{~dB}$ \\
\hline Energy consumption per year & $180 \mathrm{kWh} / \mathrm{a}$ \\
\hline
\end{tabular}




\section{Measurements and data analysis}

In order to accurately identify the vibrations we placed acceleration sensors at 47 sensitive locations. All sensors were current-fed piezoelectric transducers (IEPE/ICP). Places with non-trivial directions of movement, e.g., corners of the frame, the shell of the compressor or the cooled goods inside, were measured using triaxial sensors. To better utilize our limited measurement channels locations enclosed by two triaxially measured points were measured with uniaxial sensors. The surrounding temperature was recorded during each measurement cycle to catch variations in the viscosity of the rubber dampening elements. The refrigerator was directly placed on the floor made of concrete to ensure realistic coupling to the surroundings.

Several different actuation variants were used:

- During normal operation, the refrigerator autonomously regulates its components. The compressor and the fans operate to keep a steady temperature of $+6 \mathrm{C}$.

- Using a debugging device, interior fans are selectively activated or deactivated.

- Using the debugging device, the compressor is set to several predefined running speeds.

- Utilizing an electromechanical shaker, we actuate the shell of the compressor while the refrigerator is off the electrical grid.

\section{External sources are simulated by walking and working in the surroundings of the device.}

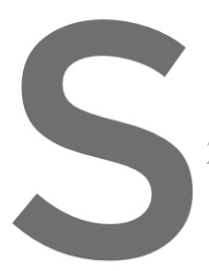

External influences are measured. The refrigerator door is opened and closed. Loading and un-

2.1

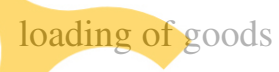

1

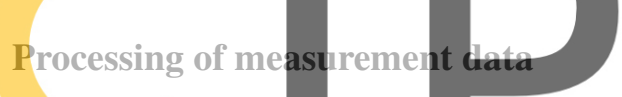

We focus our investigation on vibrations with frequencies up tc
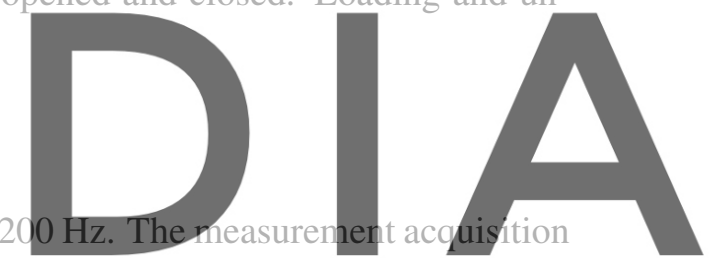

system was set to use a low-pass Bessel filter with a cut-off frequency of $1000 \mathrm{~Hz}$ and a measurement

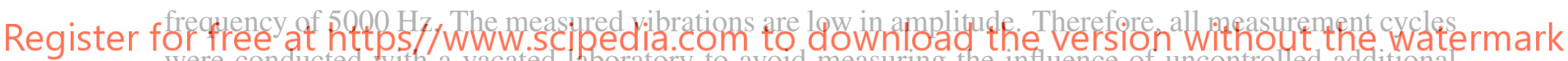
were conducted with a vacated laboratory to avoid measuring the influence of uncontrolled additional vibrations from the surroundings.

Due to the limited number of channels of the aquisition system and the limited number of sensors not all measurement points can be recorded simultaneously. However, independent measurements can be affected by different environmental effects. A noise-peak that is present in one measurement but not the other can lead to estimation error. By calculating a part-transmissibility function that specifies the relation between the point of interest and a constantly measured reference point, which is fixed for all measurements, the susceptibility to noise can be greatly reduced. The $H_{4}$-estimator $[11,12]$ was shown to improve the performance of TPA estimations and leads to robust results.

Two noisy signals $x(t)$ and $y(t)$ have the Fourier transforms $X(\Omega)$ and $Y(\Omega)$. The auto power spectral density $S_{x x}(\Omega)$ and the cross power spectral density $S_{x y}(\Omega)$ are defined as

$$
\begin{aligned}
& S_{x x}(\Omega)=\lim _{T \rightarrow \infty} \frac{1}{2 T}\left\{X_{T}^{*}(\Omega) X_{T}(\Omega)\right\} \\
& S_{x y}(\Omega)=\lim _{T \rightarrow \infty} \frac{1}{2 T}\left\{X_{T}^{*}(\Omega) Y_{T}(\Omega)\right\}
\end{aligned}
$$


where $\Omega$ is the Fourier frequency, $\mathrm{T}$ is the time interval of the measurement and the index of the Fourier transforms indicates that $X_{T}$ and $Y_{T}$ are calculated from the measurement data within this time interval T. Multiple intervals of time $\mathrm{T}$ each can be averaged to get smoother estimates of the frequency spectra. The $H_{x}$-estimators are thus calculated as follows:

$$
\begin{aligned}
H_{1}(\Omega) & =\frac{S_{x y}(\Omega)}{S_{x x}(\Omega)} \approx \frac{\sum_{i}\left[X_{i}^{*}(\Omega) Y_{i}(\Omega)\right]}{\sum_{i}\left[X_{i}^{*}(\Omega) X_{i}(\Omega)\right]} \\
H_{2}(\Omega) & =\frac{S_{y y}(\Omega)}{S_{y x}(\Omega)} \approx \frac{\sum_{i}\left[Y_{i}^{*}(\Omega) Y_{i}(\Omega)\right]}{\sum_{i}\left[Y_{i}^{*}(\Omega) X_{i}(\Omega)\right]} \\
H_{3}(\Omega) & =\frac{1}{2}\left[H_{1}(\Omega)+H_{2}(\Omega)\right] \\
\kappa(\Omega) & =\frac{\left|H_{3}(\Omega)\right|}{\max _{\Omega}\left\{\left|H_{3}(\Omega)\right|\right\}} \\
H_{4}(\Omega) & =[1-\kappa(\Omega)] H_{1}(\Omega)+\kappa(\Omega) H_{2}(\Omega)
\end{aligned}
$$

These estimations are done for each measurement point with the selected point beeing set as the $y$-channel and the reference point always being set as the $x$-channel.

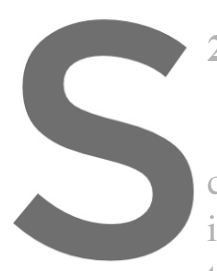

\subsection{Transfer path analysis}

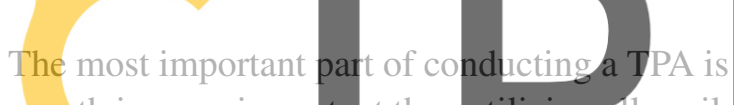

clear path is more importi

influence of different vibr
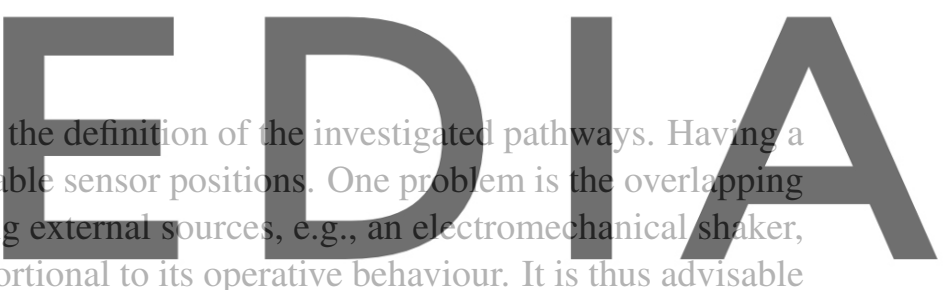

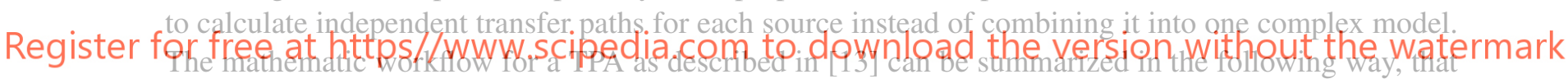

utilizes Tikhonov regularization.

If the transfer pathways are determined, the core issue of a TPA becomes estimating multiple-inputmultiple-output dependencies $\mathbf{T}$ between an input $\mathbf{X}$ and an output $\mathbf{Y}$

$$
\mathbf{X T}^{T}=\mathbf{Y} .
$$

The input $\mathbf{X}$ has $m$ channels whereas the output $\mathbf{Y}$ has $n$ channels, the width of both matrices is dependent on the number of measurement cycles or redundancies that are to be used in the calculations. Utilizing the singular value decomposition (SVD) on $\mathbf{X}$, we can obtain the parameters $\sigma_{j}, \mathbf{u}_{j}$ and $\mathbf{v}_{j}$

$$
\mathbf{X}=\mathbf{U} \Sigma \mathbf{V}^{T}=\sum_{j=1}^{m} \sigma_{j} \mathbf{u}_{j} \mathbf{v}_{j}^{T}
$$

The generalized cross validation (GCV) can then be used to select optimal parameters $\lambda$ for Tikhonov regularization 


$$
\operatorname{GCV}(\lambda)=\min _{\lambda}\left\{\frac{\left\|\mathbf{X} \mathbf{T}_{\lambda}^{T}-\mathbf{Y}\right\|^{2}}{m-n+\sum_{j=1}^{m}\left(\frac{\lambda^{2}}{\lambda^{2}+\sigma_{j}^{2}}\right)}\right\}
$$

These optimal values of $\lambda$ can then be inserted into

$$
\mathbf{T}_{\lambda}^{T}=\sum_{j=1}^{n} \frac{\sigma_{j}^{2}}{\lambda^{2}+\sigma_{j}^{2}} \frac{\mathbf{u}_{j}^{T} \mathbf{Y}}{\sigma_{j}} \mathbf{v}_{j}
$$

and with our previously calculated SVD-parameters, the estimate of the transmissibility function matrix $\mathbb{T}_{\lambda}^{T}$ is fully defined. These calculations then have to be applied in iteration for every considered frequency in the frequency range. The input matrix $\mathbf{X}$ and the output matrix $\mathbf{Y}$ are varied according to the previously determined pathways and are filled with the respective sensor data to calculate all necessary transmissability functions. Finally, the results are visualized in false color plots as seen in figure 2.
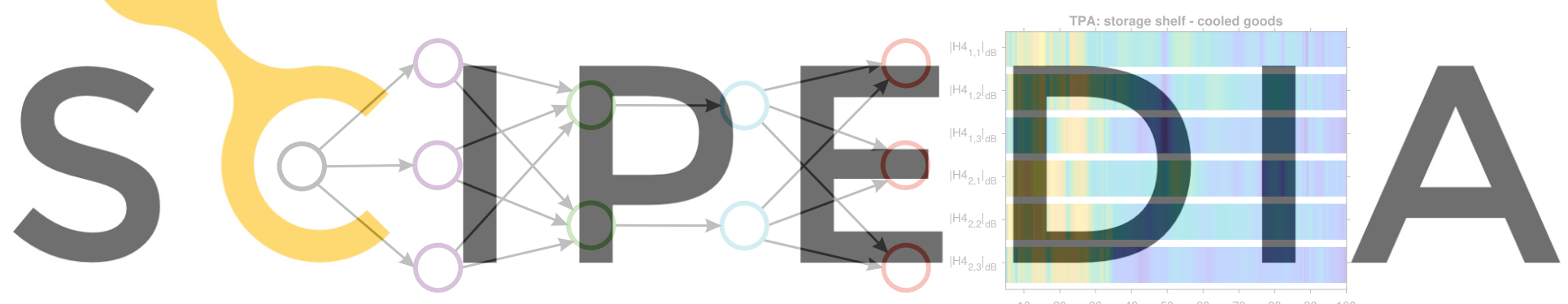

Register for free at https//wwww.scipedia.com to download the version without the watermark

Figure 2: A possible pathway for a TPA (left) and an examplary result of the calculations (right) that visualizes the transfer paths between the wooden storage shelf and the cooled goods. The yellow colored areas in the plot indicate frequencies that are only weakly damped within this sub-path.

\subsection{Operative deflection shape analysis}

The ODS analysis is used to visualize the spatial movement of the investigated object and is similar to the modal analysis. It works best in static operation conditions but if the internal excitation is too weak, an additional external excitation can be necessary. Using a shaker at static frequency one can calculate the Fourier transform of all signals for the chosen frequency. Variation of the excitation frequency enables the calculation of a spectral frequency response that should be refined through the previously mentioned $\mathrm{H}_{4}$-estimation process of eq. (2). The positions and the measuring directions of all sensors are known and can be input into a spatial model of the device. The calculated transmissiblity functions from the reference point can then be visualized as a spatial distortion that is dependent on the frequency 
of the excitation. Using an arbitrary scaling factor all sensor positions are moved according to this transmissibility function in a sinusoidal way. The amplitude indicates how big this movement should be and the phase is a delay. The frequency of the visualized oscillation is not relevant.

The resulting images can be put side by side or an animated video can be produced that shows the movement of the device at this frequency. If the investigated frequency is a natural frequency of the device, distinct modal shapes will be visible. Otherwise, the modal shapes will overlap into the cumulative behaviour. Figure 3 shows a high mobility of the shelves and slight bending of the entire frame. This can be used as the basis for future design decisions.

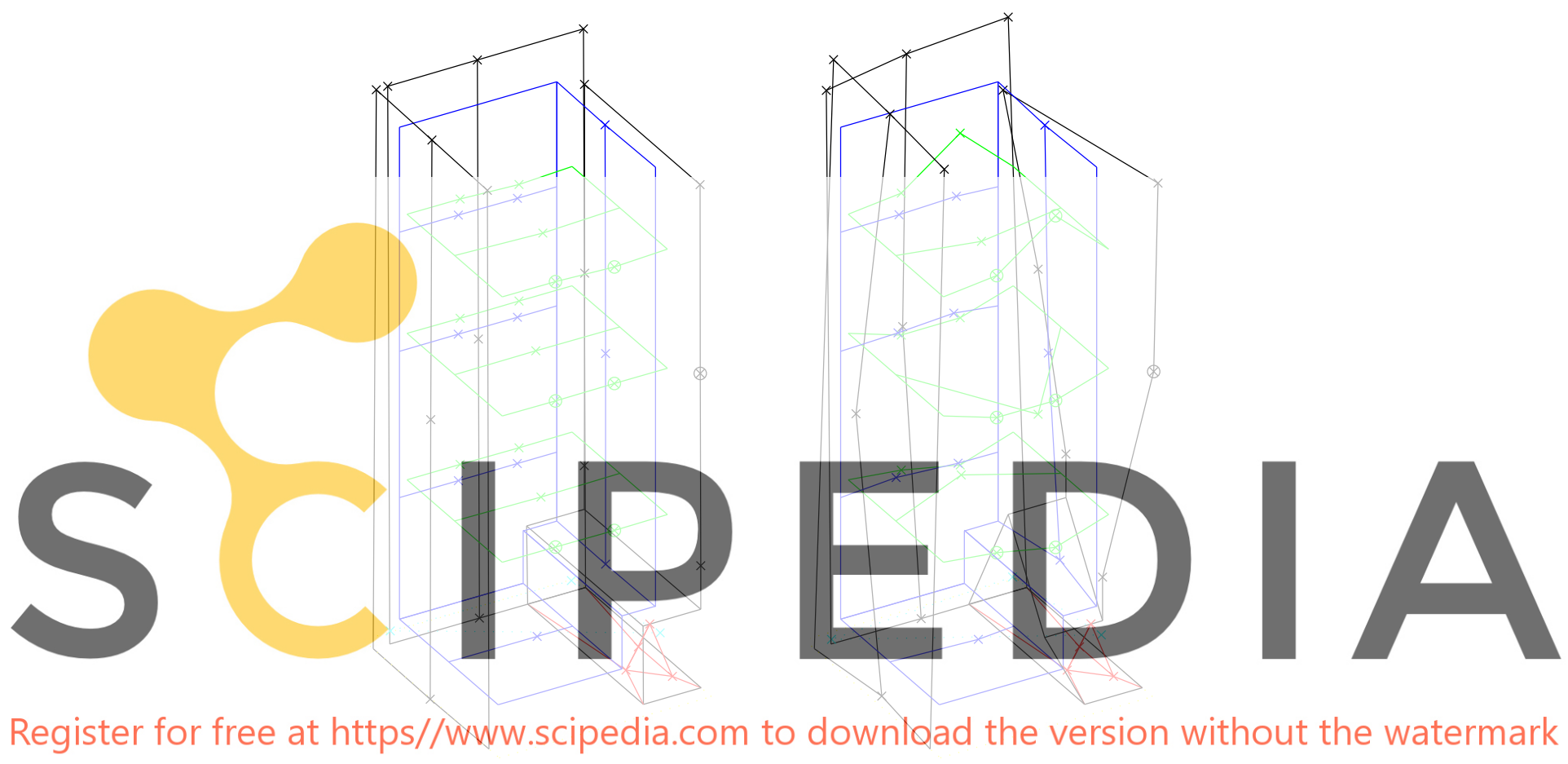

Figure 3: Visualization of a ODS analysis at a specific frequency. All points are measured and the lines connect the sensors to aid visualizing the original form of the device. High mobility of points indicates low stiffness and low mobility indicates that the points are only weakly excitated by the measured source. This visualization can be helpful for future design desitions, e.g., stiffening of mobile areas.

\section{Identification and quantification of vibrational sources}

In order to prioritize the development time efficiently the impact of different vibrational sources is quantified. The considered sources can be categorized as follows:

- internal active sources

- compressor

- fans 
- additional features, e.g., ice makers

- internal passive sources

- movement of the cooling fluid

- airflow due to the fans

- external sources

- base excitation through the surroundings

- people walking near the refrigerator

- work being done in the vicinity of the refrigerator

- opening of the refrigerator door

- loading and unloading of the refrigerator

RMS (root mean square value) measurements of the vibration of the cooled goods are the simplest way to quantify the impact of different vibration sources but these numbers are not to be used directly. The different sources should be identified as independent as possible. The following workflow was used.

Several acceleration sensors are evaluated, one is placed at the compressor and the others are placed on the cooled goods. For the first measurement the refrigerator was off the grid and the laboratory was vacated. The base excitation through the surroundings was measured including the measurement noise of the sensors. Then we measured the impact of different isolated external sources. Next the refrigerator

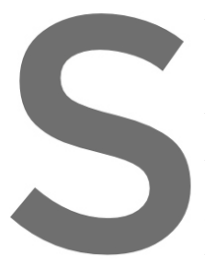
was plugged in and switch selective activation of the varying frequencies. Fina ational conditions and to we used the measured data frr
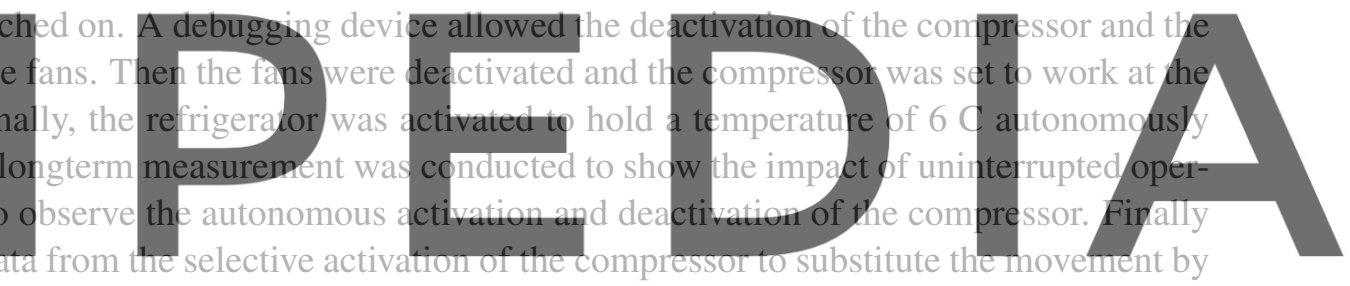

externally exciting the compressor with the electromechanical shaker

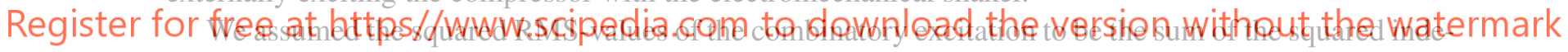

pendent excitation sources. This allows the reduction of the operational RMS-measurement by the base measurement and enables us to independently quantify the impact of the cooling fluid moving through the pipes by comparing the active operation of the compressor with the substituted shaker movement.

Now that all sources are quantified it is necessary to make assumptions. How will the behaviour average over 24 hours? How often is the door opened? How often will someone walk by with light/heavy steps? Opening the door resulted in oscillating spikes of $8500 \mathrm{~mm} / \mathrm{s}^{2}$ that were fully damped within $2 \mathrm{~s}$. The impact of these numbers highly depends on the rate of occurrance. We assumed that within 24 hours the door is opened 10 times, for 5 hours there is light work in the surroundings with people walking lightly and for 15 minutes each day there is heavy lifting in the vicinity. These assumptions can vary based on the specific use case.

\section{Results}

Utilizing the discussed methods, we calculated the impact of the differing vibration sources. The exact numbers cannot be disclosed in this paper but using this method, we could prioritize future development 
and suggest likely solutions. In figure 4 we see a transmissibility function for vibrations that are excited by the compressor. It is shown that this device has several natural frequencies in areas that are suitable for compressor operation. Operation within the marked frequency intervals hardly excites the cooled goods, minimizes the exposure to vibration and has the additional potential to reduce the audible noise.

Figure 5 shows a natural frequency of the condenser on the back of the refrigerator. This condenser is mounted on a lattice made of steel wire that is held on place by a sturdy frame. A quick analytical approximation of this structure can be made using the formulae for circular Euler-beams made of steel.

$$
\begin{aligned}
\omega_{0} & =2.55 \cdot 10^{4} \frac{R}{L^{2}} \\
\omega_{n 1} & =k_{1} \omega_{0}
\end{aligned}
$$

The beams have a diameter of $R=1.4 \mathrm{~mm}$ and a length of $L=1280 \mathrm{~mm}$. For fixed supports the multiplicative faktor is $k_{1}=2.267$. Evaluating equation (7) the expected natural frequency is located at $\omega_{n 1}=24.71 \mathrm{~Hz}$. This closely aligns with the results of our measurements which are shown in figure 5 . A change in the lattice dimensions could shift this natural frequency and influence the transmissibility of the overall system.

Utilizing the data gained from the TPA and ODS analysis areas of interest have been identified that will be addressed in design reviews. By combining these optimizations the cooled goods will be exposed to significantly reduced amounts of vibrations. These improved conditions facilitate long term storage without degradation of quality.
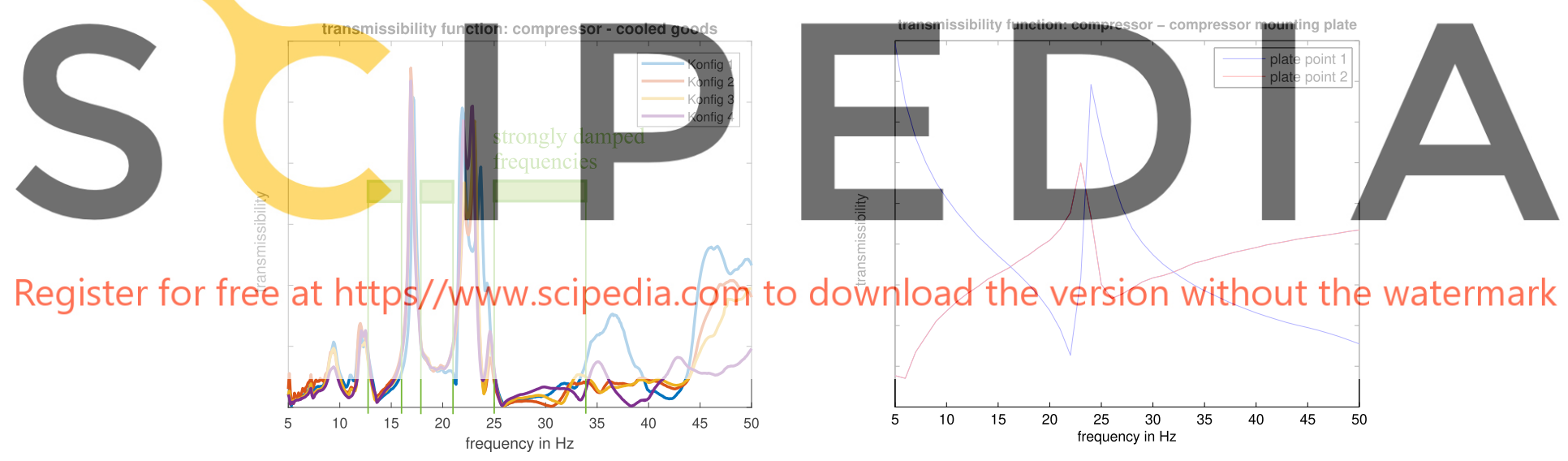

Register for free at https//www.scipedia.com to dowñ

Figure 4: Examples of acquired transmissibility functions and their interpretation. Left: transmissibility from the compressor to the cooled goods for repeated masurements. The refrigerator has natural frequencies at $17.1 \mathrm{~Hz}$, $22.2 \mathrm{~Hz}$ and around $23.5 \mathrm{~Hz}$. Operation in the strongly dampened frequency intervals marked in green hardly excites the cooled goods. Right: transmissibility function from the compressor to points on the compressor mounting plate. This function describes the effectiveness of the grommets that isolate the compressor from the steel frame. The grommets show a natural frequency around $24 \mathrm{~Hz}$.

\section{Conclusions}

In this paper, we investigated various methods to analyze vibrations within multi-body systems. We have listed possible workflows and explained the applicability of the methods for different circumstances. 

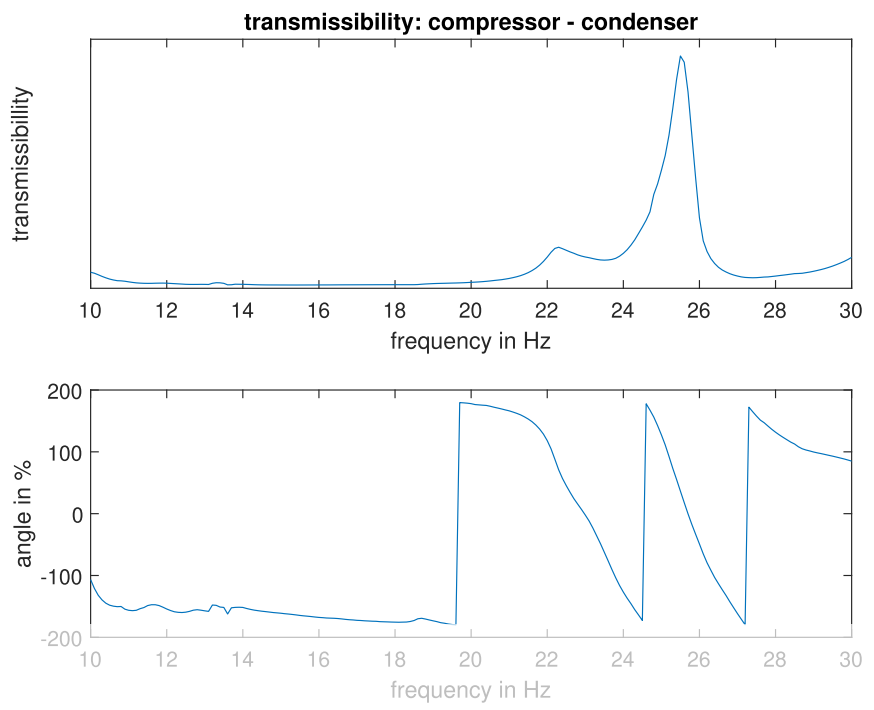

Figure 5. Transmissibility from the compressor to a point on the condenser at the back of the refrigerator. A natural frequency at $24.7 \mathrm{~Hz}$ is clearly visible.

Using our investigated refrigerator model we have shown clear results of our measurements and suggested immediate solution

vibration. The multi-later highlights areas of inter

Acknowledgments
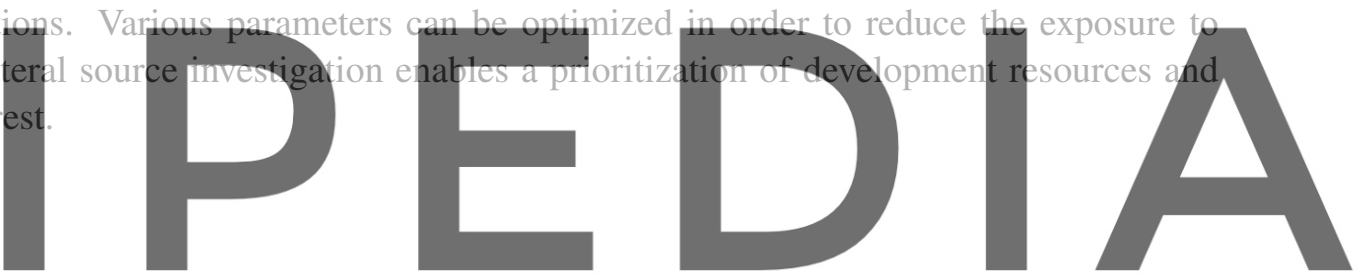

This work was supported by the Österreichische Forschungsfördergesellschaft mbH (FFG) (No. 18819992)

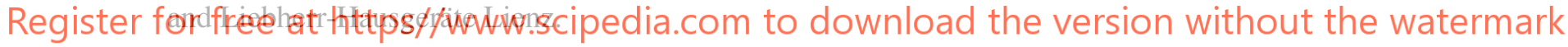

\section{REFERENCES}

[1] Takushima, A., Shinobu, Y., Tanaka, S., Eguchi, M. and Matsuke, K. Fan noise reduction of household refrigerator. (IEEE) Transactions on Industry Applications. (1992). 28 287-292

[2] Tojo, K., Machida, S., Saegusa, S. and Hirata, T. Noise Reduction of Refrigerator Compressors. International Compressor Engineering Conference. (1980).

[3] Bailif, E.A. and Laughlin, J.P. Analysis and evaluation of noise in household refrigerators. (IEEE) Electrical Engineering. (1959). 78 646-650

[4] Çelikkan, O. and Erol, H. Vibroacoustic Analysis of a Refrigerator Freezer Cabinet Coupled with an Air Duct. Advances in Acoustics and Vibration. (2017)

[5] Kuyumcuoğlu, A. and Sakalli, Ö. Investigation of vibration transmission properties of compressor grommets in domestic refrigerators. Inter-noise 2014. (2014) 
[6] Sayer, A. and Greussing, D. Anwendung der Interface-Mobilitäten auf einen Kühlschrankkompressor. Fortschritter der Akustik - DAGA 2016. (2016). (in german)

[7] Chung, H.J., Son, J.H., Park, E.Y., Kim, E.J. and Lim, S.T. Effect of vibration and storage on some physico-chemical properties of a commercial red wine. Journal of Food Composition and Analysis. (2008). 21:8. 655-659.

[8] Tao, Y., Garca, J.F., Sun, D.W. Advances in Wine Aging Technologies for Enhancing Wine Quality and Accelerating Wine Aging Process. Critical Reviews in Food Science and Nutrition. (2014). 54:6. 817-835.

[9] Crandles, M., Wicks-Müller, M., Schuessler, C. and Jung, R. The Effect of Simulated Transportation Conditions on the Chemical, Physical and Sensory Profiles of M"uller-Thurgau and Scheurebe Wines. Journal of Food Science and Engineering (2016). 6

[10] Nikolaev, N.I., Liu, Y., Hussein, H. and Williams, D.J. The sensitivity of human mesenchymal stem cells to vibration and cold storage conditions representative of cold transportation. Journal of The Royal Society Interface. (2012). 9 2503-2515

[11] Kühlert, H., Markert, R., Witfeld, H. Identifikation von Übertragungsfunktionen durch Mehrpunkterregung mit breitbandigen korrelierten Signalen. Schwingungen in der Fahrzeugdynamik. Vieweg+Teubner Verlag. (1991). (in german)

[12] Hörtnagel, W.A., Plagg, S. and Dohnal, F. Harmonic Transfer Path Analysis of a Wine Refrigerator. DSTA 2019, December 2-5. Łódź, Poland. Springer Proceedings. (2020). (in press)
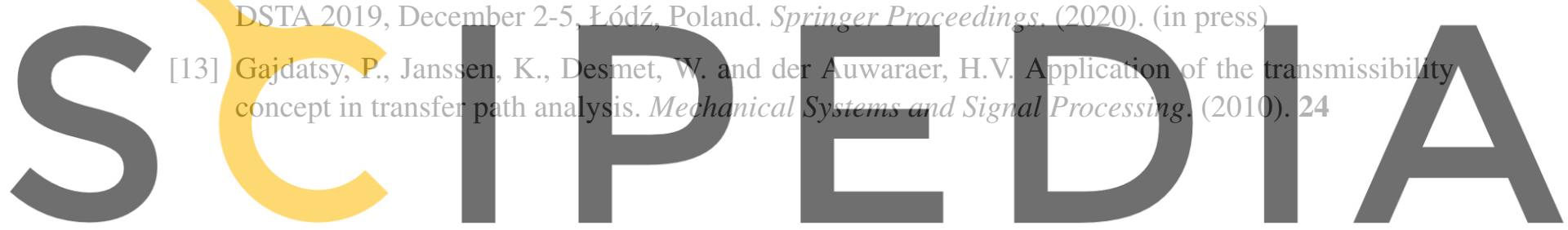

Register for free at https//www.scipedia.com to download the version without the watermark 